
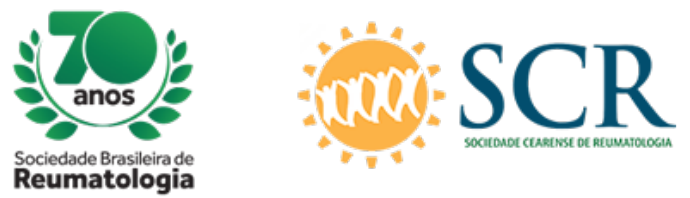

\title{
SUBJECTIVE FATIGUE AND SKELETAL MUSCLE ARE ASSOCIATED WITH MUSCLE METABOLIC PROFILE IN RHEUMATOID ARTHRITIS
}

Thales Hein da Rosa (Ufrgs, CANOAS, RS, Brasil), Marianne Schrader de Oliveira (Ufrgs, Porto Alegre, RS, Brasil), Paulo Vinicius Alabarse (UCSD, Estados Unidos), Rafaela Cavalheiro Espírito Santo (Ufrgs, Porto Alegre, RS, Brasil), Jordana Miranda Souza Silva (Ufrgs, Porto Alegre, RS, Brasil), Stephen Pete Young (University of Birmingham, Grã-Bretanha (Reino Unido)), Ricardo Machado Xavier (Ufrgs, Porto Alegre, RS, Brasil)

\section{BACKGROUND}

Rheumatoid arthritis (RA) is an autoimmune disease characterized by chronic inflammation and represents an important cause of mortality and incapacity. Besides, RA is also associated with metabolic comorbidities, such disorders in lipid and glucose metabolism and cachexia. Since the metabolomic profile is known to vary in response to different inflammatory conditions, metabolome analysis could provide patterns to correlate with clinical parameters and potentially become biomarkers which might improve diagnosis of RA patients.

Objective: Verify the correlation between metabolic profile and body composition and disease activity in AR patients.

\section{MATERIALS AND METHODS}

Seventy-nine RA patients, according to ACR/EULAR 2010 classification criteria, aged between 40 and 70 years, were recruited characterized and followed for 12 months. Patients disease activity was measured by Disease Activity Score-28 (DAS-28) with erythrocyte sedimentation rate (ESR). Also, was made the fatigue assay by Functional Assessment of Chronic Illness Therapy (FACIT). Body composition of the patients was verified by appendicular lean mass index (ALMI) and values were collected by total body dual-energy x-ray absorptiometry (DXA). Finally, metabolome analysis was made using the Nuclear Magnetic Resonance spectroscopy (NMR) followed by characterization of 48 metabolites identified as involved in metabolic changes in disease development.To statistics, Frequency analysis, Pearson Correlation and Multivariate data analysis with orthogonal projections to latent structures (OPLS) was performed and a statistical significance was considered as $p<0.05$.

\section{RESULTS}

In the population characterization, was observed a majority of women (86,7\%), with mean age of 56 years old. Also, $80 \%$ of these patients present anti-citrullinated cyclic peptide (CCP) and positive for Rheumatoid Factor (RF). In twelve months period, there was no variation of DAS28 measurement (baseline: 3,8 , after 12 months: 4,0). There was no significant correlation between the metabolome pattern and changes in DAS28 score $(p>0.05)$. In metabolome analysis, was observed a significant increase of metabolites involved in muscular metabolism: methyl-histidine, L-serine, creatinine and urea. Fatigue was negatively correlated with L-serine/creatinine ( $r$ : - 0,4, $\mathrm{p}<0.001)$. Appendicular lean mass index (ALMI) also showed a difference which correlated with the increase of urea and creatinine $(r: 0,3, p<0.019)$. 
The potential biomarkers might be interesting to associate RA inflammatory profiles and activity with metabolic comorbidities present in disease. Our findings suggest that urine metabolome analysis may be an interesting approach to monitoring rheumatological disease related to fatigue that could be more explored in future trials. 